\title{
Patient Monitoring System for Easy Supervision using LabVIEW.
}

\author{
Prajwal M J, Prajwal M
}

\begin{abstract}
This study is aimed to develop a self-managing application for patients. Here, a novel model of an automatic pill reminder that can allay the inconsistency or uncertainty is presented. This application is useful in taking prescribed medications of the right dosage at the exact specified time guided by the medical practitioner. Hence, believed to shift from some particular approaches that are most voluminously resting on the human memory to automate with negligible oversight. So as to relieve people from human miscalculations of giving wrong pills at the wrong clock in the wrong amount.
\end{abstract}

Keywords: Medication adherence, eHealth, Elderly Healthcare.

\section{INTRODUCTION}

APILLreminderisanyapplicationthatremindsitsuserstotakeme dicationsatproperspecifiedclock [1].Medications are considered to be one of the most important healthcare technologies for improving the longevity and quality of life. The interpretation of quality health care is abided with provision of assistance in a timely manner.[2]. Today, a great amount of resources including money, time and human interference are required for good 24-7 health-care monitoring and directory facilities. This added with their refutable tendency of the human memory in failing to recall any specifications can result in consequential irregularities, critical situations and melancholy. Repeatedly, we are unable to assimilate the harm we apply or leave it midway altogether, or may also abominably take the wrong dosage. While computerization and modern technology has backed the field in some of the key regions to remove human flaws and have achieved a desired grade of efficacy, taking pills at the right time is still not visualized to be an area which could be coordinated or monitored by the modern applied science. So, an attempt has been made a cost-efficient device that is easily accessible and increases medical adherence.[3] In an internet driven world an application that uses Noor minimal internet requirements can be a good appeal. Such an application can prove it a 1 in the rural section soft he country where internet access is still hard to find.

\section{MOTIVATION}

The inclination to design such an application has been picked up from observing the elderly around us. Sometimes misremember to take proper pills prescribed by the doctor, thus missing out medications in the non-attendance by a family member or an appointed nurse.[4] For such scenarios

Revised Manuscript Received on December 09, 2019.

Prajwal M J, Department of Electronics \& Communication, RNS Institute of Technology, Bengaluru, India. Email: mj.prajwal1 @ gmail.com

Prajwal M, Department of Electronics \& Communication, RNS Institute of Technology, Bengaluru, India. Email: prajwalmahaveera@gmail.com

as well a proper medication reminder tool will come handy. Generally, health service centers also exhibit lack of attendance at times, which exacerbate or intensify the described situation. All these circumstances gave the encouragement to bring in a simple and feasible solution that is easily available and operable for the aged and the ailing. The aim was also to provide easy communication to them.

\section{SOFTWARE SPECIFICATIONS}

Laboratory Virtual Instrument Engineering Workbench (LabVIEW) is a system-design program. It is a graphical programming language named ' $G$ ' and a building domain from the National Instruments. This is visual coding language is used by defining the relations and networks among objects in graphical diagram. This language is dissimilar and unusual than any other coding language in various manners. To begin with, the programs in this software are linearly executed. Only after completely executing the previous command, the execution passes on to the next step. A graphical block of code can only be executed when its inputs are defined or calculated previously in the program. A novelty which is not naturally found in the traditional text-oriented programming languages called the sequence structure, that permits linear execution of certain sections of the block code along with the usual structures like the while and for loop, case structures are provided for simple and efficient programming or realization of the designs. This can be seen to be a very handy feature in situations identical to those when the code needs to primarily send some instruction to a particular equipment, and followed by the wait for a moment and scan the information attained from that equipment.[5] This can be seen to be a very handy feature in situations identical to those when the code needs to primarily send some instruction to a particular equipment, and followed by the wait for a moment and scan the information attained from that equipment.[6]

\section{WORKING OF THE PROGRAM}

\section{A. PATIENT'S INTRODUCTION}

At the beginning, the basic information of the patient such as name, reference number, consultant doctor, department etc. are taken as input strings for any reference as shown in figure 1 .

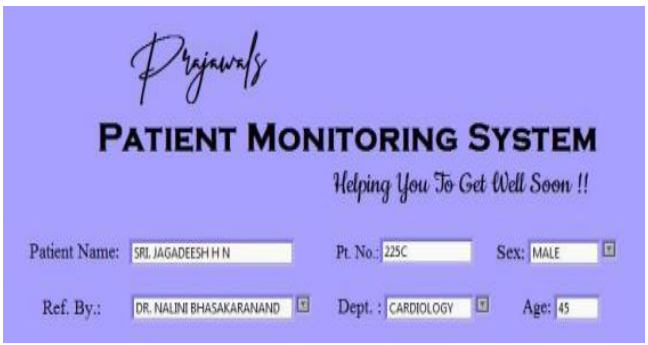

Fig. 1. Patient's information 


\section{Patient Monitoring System for Easy Supervision using LabVIEW}

\section{B. INPUT MEDICINES}

Figure 2 and 3 shows how the inputs are taken in the back end of the program. In this block ' $n$ ' number of medicines with their dosages are taken as input. Names of the pills are taken in as strings using combo box control;

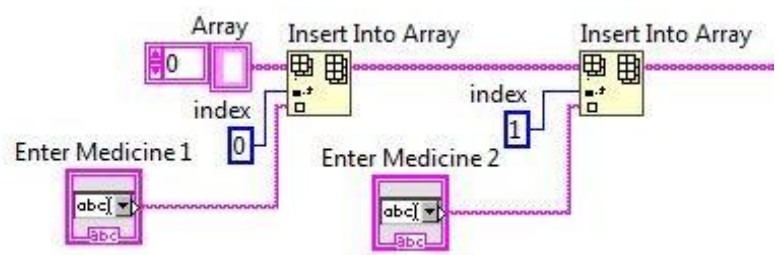

Fig. 2. Taking input of Medicines

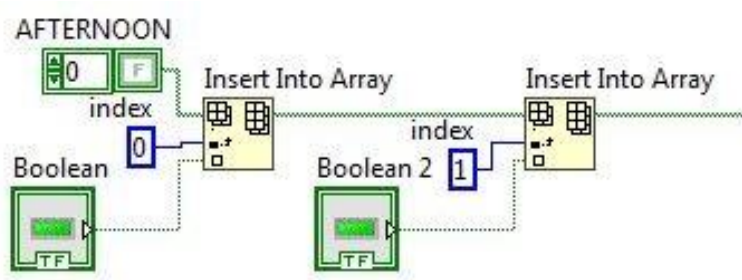

Fig. 3. Taking input of dosages

dosages are taken in Boolean format and they are converted to array using insert into array function.

\section{COMBO BOX CONTROL}

Combo Box control is used to take in and generate a set of those strings that are often used, such as pull-down menu. Combo box controls are different from ring and enum controls because they output data as strings instead of numeric. As you type an input into the combo box during the run time, the software selects the shortest first parameter from the pre-defined set of strings that begins with the letters typed. If it finds no alike parameters that match the letters typed, the control still allows undefined string values, given that the defaults are modified. Figure 4 shows the front panel and the drop-down box.

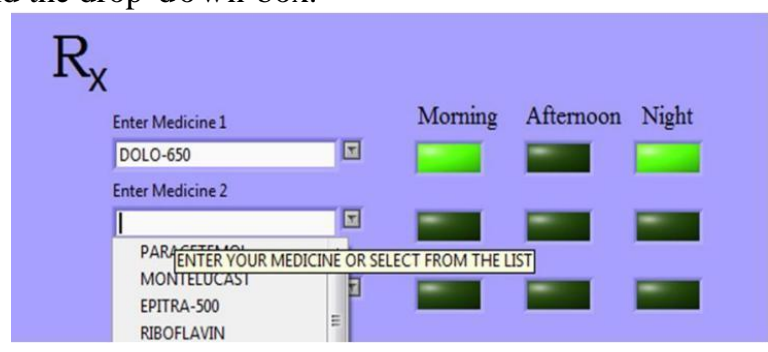

Fig. 4. Input layout in front panel.

\section{CREATING CLUSTER}

The inputs are then bundled into a single wire using array to cluster function which transforms a one-dimensional array into a cluster of elements which are of the same type as the elements of the array. The figure 5 shows the same. This is done to enhance the readability and debugging process.

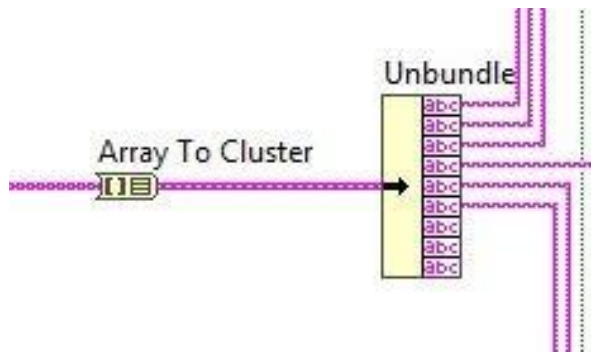

Fig. 5. Array to cluster.

Now this cluster passed on into the further loop and separated using the in-built function unbundle. It slices the given cluster into the respective original elements. When this function receives a cluster through a wire, it rescales accordingly to indicate nodes for each member in the function. A set of terminals that corresponds to the indicators, present the default data types for this discrete function.

\section{E. TIME STAMP}

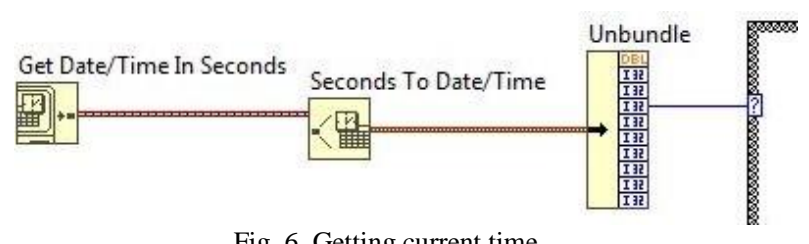

Fig. 6. Getting current time.

Figure 6 shows the block diagram of the time stamp. Get time in seconds is the function we use here to get the current time from the system. This function gives out a timestamp of the present time. The first function gives the number of seconds that has passed from 12 AM, Friday, Jan 1 of 1904. This data will be passed to the Seconds to Date/Time converter, which calculates the present time and date using the previous data gives out the value as a cluster. This cluster is unbundled and the time is acquired. This is used to confirm if the time is up for the pills to be consumed.

\section{F. CASE STRUCTURE}

The case structure has one or more sub diagrams, or cases defined to be executed at some particular conditions. The second type of input into the case structure will be the case selector. The case selector is any condition that is to be checked continuously for trueness, when the condition matches that particular block of code or diagram is to be executed. The case structure inputs dosages and names as their primary inputs which are processed in the structure. The check timings for the dosage are taken as selector labels as specified by the medical practitioner. When the current time equals the specified time, the structure is executed. Now each medicine is checked for its dosage and built into an array using build array function and shown on monitor. 


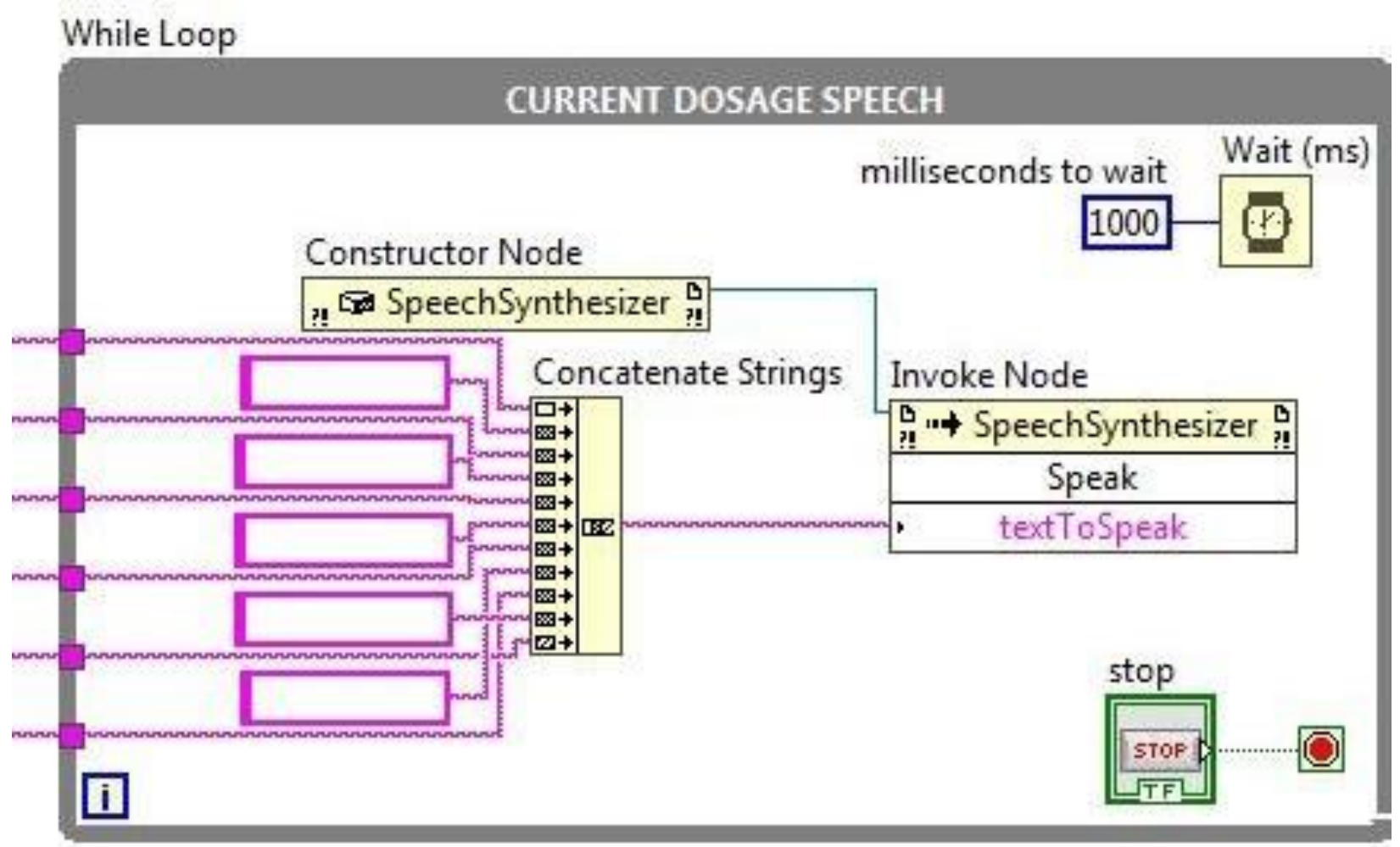

Fig. 7. String to speak

\section{G. SPEECH SYNTHESIS}

Now the strings are taken in from the previous loop that match the timings and are put into concatenation. To differentiate the names, they are concatenated with empty strings in between. Now the concatenated string is passed to invoke node. Figure 7 is the block diagram of the abovementioned process.

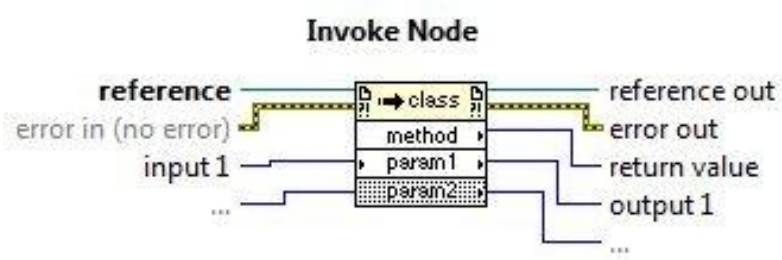

Fig. 8. Invoke node

Figure 8 shows the inputs and outputs of the invoke node. It enforces a process or operation on a particular reference. Almost all methods hold associated specifications .A reference is passed to the invoke node using constructor node. It generates a sample of .NET object. The constructor from which a .NET object is to be established is identified by this node. The name of the medicines which are obtained after concatenating them between empty strings are made to speak using the speaker of the system. This speech is repeated until the stop button on the monitor is checked. Then the loop execution ends and the system stops alarming. Using the above specified functions provided in the LabVIEW software we developed the Patient monitoring System Program.

\section{FLOW CHART:}

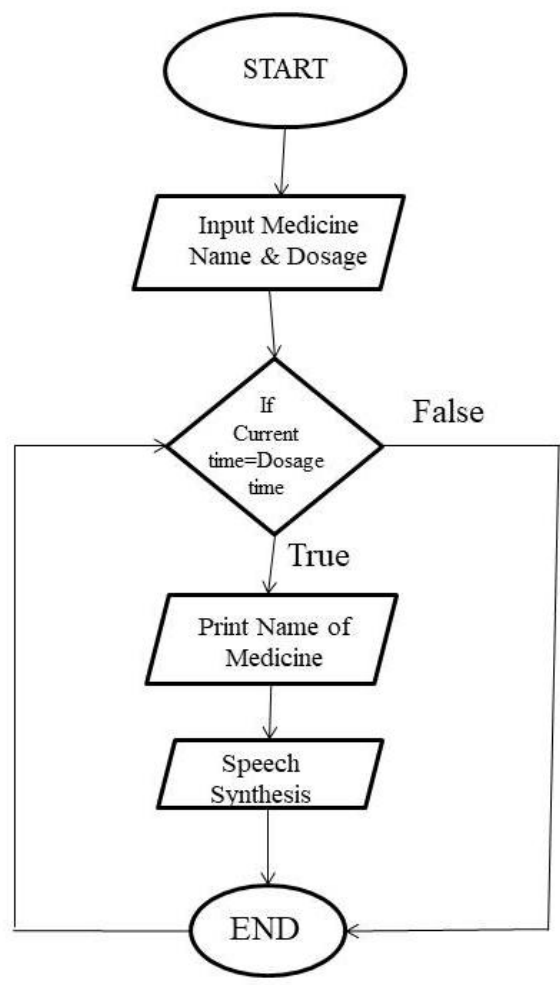

Fig. 9. Flow Chart. 


\section{NEED AND DISCUSSIONS}

A recent study by the United Nations articulates that the all- around population of senior citizens by the year 2030 is anticipated to have exceeded that of the minors (1.41 billion against 1.35 billion). The number of people aged beyond 60 might be more than the number of people aged between 10-24 years. That is 2.10 billion against 2.0 billion of the population which is illustrated in figure 10 . The life expectancy of the people globally is rising as well. Between 2017 and 2050, the population aged 80 years or over is expected to triple in number, proliferatingfrom 137 millionto a huge425million.[7]

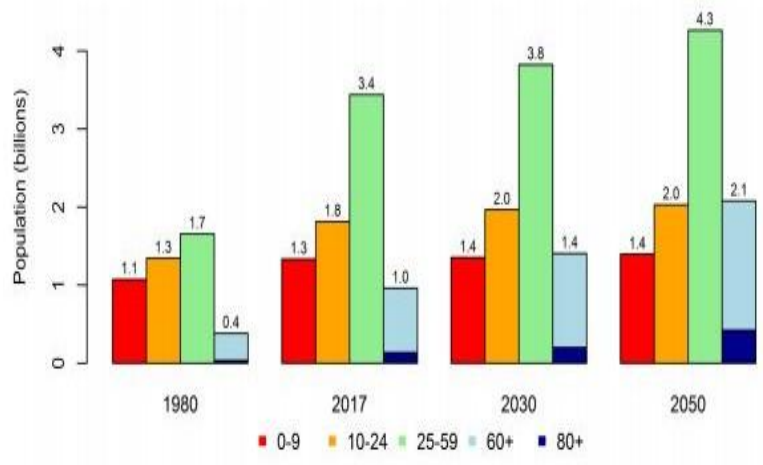

Fig. 10. Global population of the age group, in 1980, 2017, 2030 and 2050

The intensification of recurrent medical circumstances, increase in inpatients, and further unnecessary health care costs can occur due to inadequate medication obedience amidst the section of older adults. As hospitals progressively visions to maintain good health of the population, one approach to increase overall cohesion with medications is the use of technology in every patients' homes as well. Missed pill consumption, taking extra pills, and fetching wrong dosages and many other medication errors, can be resolved by including the successful interface with modern technology.[8]

\section{ADVANTAGES}

Our current LabVIEW program size approximately $45 \mathrm{~KB}$ and taking into considerations of all the future updated additions to the program that are specified in the future scope, the size of the program is not expected to reach beyond $100 \mathrm{~KB}$. This means the LabVIEW program can be easily run in any system with any basic specifications without any high-end requirements. The real time implementation of this application is highly cost efficient and easy to maintain.

\section{RESULTS}

This application helps in keeping track of day to day medicinal practices and alleviates human administration and oversight. It is a simple and smooth application thus adds in as a prosperous benefit for the aged and the ailing, and caretakers for the suffering and the old folk. This study tends to find its application in the masses at every house or health center that may regularly have medical oversight issues thus can be advertised as an adequate solution to the masses. The application results desired at the specified time is shown in figure 11 .

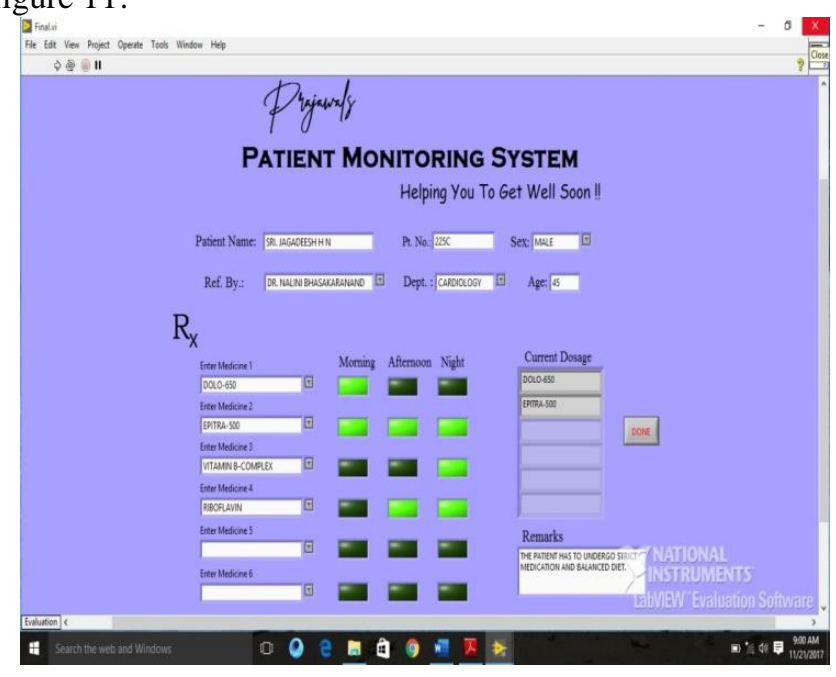

Fig. 11. Output display on the monitor and speech

\section{FUTURE SCOPE}

This application can be further built by interfacing the GSM module. The SIM 800 L / SIM 900 AT module which supports quad-band GSM/GPRS network, available for SMS message data remote transmission and GPRS can be interfaced to this program.[9] The application can ask the user if the user wants the application to remind the pills by displaying it on the monitor or by sending to the user's phone an SMS notification or a reminder. It can be made to ask the user whether he/she has taken the pill or not after reminding. If the user reports that the pill has not been taken, the application reminds the use regain after few minutes to take the medication. And it can also be made to report the person who is taking care of the patient whether he has taken the medication or not. Using these data created about the intake of pills, the application can be made to record the same and write it to a file for any future reference. Some other attributes like blood pressure, heart rate and body temperature etc. of the patient can be recorded time-to-time by interfacing suitable sensors with the program and storing it in the database and showing it on the output screen.

The application can provide the option to place an order for the medicines on the online shopping portals automatically by calculating the quantity of those using the dosage and the number of days prescribed by the medical practitioner to the medicines.

\section{CONCLUSION}

Using the above needful specifications, a reasonable, economical and an advantageous exemplary model of an automated pill reminder is created using LabVIEW. For convenience a separate array is built so as the user or the care taker is accurately reminded of the exact pill to be consumed in the right quantity, at the right time without human guidance. For the future medical consultation, a useful database or a record could be built by recording the pill intake timings and the regularity of the patient. This simple application can be a handy,

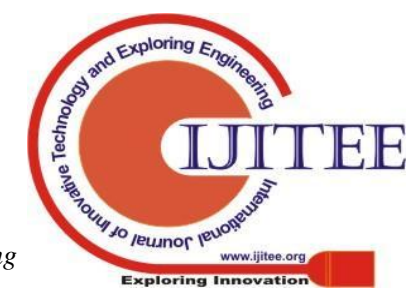


user friendly choice for families where the members might have their job time constrains or other commitments are necessitated to get a nurse for the person with medical botheration.

\section{REFERENCES}

1. "Pill reminder" https://en.wikipedia.org/wiki/Pillreminder

2. Healthcare Surveys- Employee \& Patient Satisfaction Surveys https://www.nbrii.com/products/healthcare-surveys

3. Ameta, Deepti \& Mudaliar, Kalpana \& Patel, Palak. (2015) Medication Reminder and Healthcare-An Android Application .International Journal of Managing Public Sector Information and Communication Technologies. 6. 39-48.10.5121/ijmpict.2015.6204.

4. Billingsley, Luanne, and Ann Carruth. "Use of technology to promote effective medication adherence." The Journal of Continuing Education in Nursing 46.8 (2015): 340342

5. http://www.ni.com/getting-started/labview-basics/environment

6. Introduction to $\mathrm{G}$ programming language http://docbook.rasip.fer.hr/rip smil/14G/Example/html/gintro.html

7. "World Population Ageing- theUnited Nations." https://www.un.org/en/development/desa/population/publications/pdf/ ageing/WPA2017

8. McLaughlin, Eric J., et al."Assessing medication adherence in the elderly.” Drugs and aging 22.3 (2005):231-255.

9. Jabeena, Afthab \& Sahu, Animesh \& Roy, Rohit \& Basha, N.. (2017) Automatic Pill Reminder for Easy Supervision. https://arxiv.org/abs/1711.09739

\section{AUTHORS PROFILE}

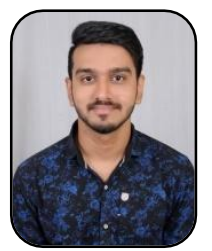

Prajwal M J holds bachelor degree in Electronics and Communication Engineering in RNS Institute of Technology, Bengaluru under VTU Belagavi. He has presented various projects in Open-House project Expos and few of them have been selected as Best Project in their respective categories. He has published a paper on Wireless Power Transmission using DC Tesla Coil in the National Conference conducted by IETE on 2018 . His areas of interests include adaptations of technology in social applications, creating chatbots, python and LabVIEW programs.

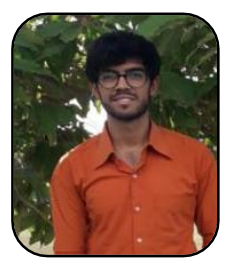

Prajwal $\mathbf{M}$ is obtained his bachelor degree in Electronics and Communication Engineering in RNS Institute of Technology, Bengaluru under VTU Belagavi. He has presented various projects in Open-House project Expos and few of them have been selected as Best Project in their respective categories. His areas of interests include adaptations of technology in Social impact, coding sections, implementing new ideas into IOT projects and LabVIEW programs 